\title{
Hille-Yosida type theorems for local regularized semigroups and local integrated semigroups
}

\author{
by \\ Sheng Wang Wang (Nanjing)
}

\begin{abstract}
Motivated by a great deal of interest recently in operators that may not be densely defined, we deal with regularized semigroups and integrated semigroups that are either not exponentially bounded or not defined on $[0, \infty)$ and generated by closed operators which may not be densely defined. Some characterizations and related examples are presented. Our results are extensions of the corresponding results produced by other authors.
\end{abstract}

1. Introduction. Let $X$ be a complex Banach space and let $A$ be a closed linear operator on $X$. Many physical problems may be modelled as a first order abstract Cauchy problem:

$(\mathrm{ACP}, T) \quad\left\{\begin{array}{l}u^{\prime}(t)=A u(t), \quad t \in[0, T), \\ u(0)=x,\end{array}\right.$

where $0<T \leq \infty$. The first order abstract Cauchy problem $(\mathrm{ACP}, \infty)$ is well-posed if $A$ is densely defined and generates a strongly continuous semigroup. But the theory of strongly continuous semigroups does not apply to the case when $A$ is not densely defined. Examples of this situation appear in population models. When $A$ is densely defined, if it does not generate a strongly continuous semigroup, then $(\mathrm{ACP}, \infty)$ is still ill-posed. Examples are the backwards heat equation, the Schrödinger equation on $L^{p}, p \neq 2$, etc.

To deal with the ill-posed abstract Cauchy problem, two approaches have been established and achieved great success. Davies and Pang [4] introduced the concept of exponentially bounded $C$-semigroups (which is equivalent to the concept of $R$-semigroups of exponential growth introduced by Da Prato [3]). About the same time, Arendt [1] introduced the concept of integrated semigroups (for the exponentially bounded case, see also [11]).

As regards the theory of strongly continuous semigroups and cosine operator families and their applications, in addition to the monograph [6], the

2000 Mathematics Subject Classification: Primary 47D06; Secondary 47B40. 
interested reader may consult the monographs [5] and [12]; for the theory of regularized semigroups and integrated semigroups and their applications, the monograph [8] is of importance.

The purpose of this paper is to present several Hille-Yosida type theorems for local regularized semigroups and local integrated semigroups generated by closed operators which may not be densely defined, and consider the local abstract Cauchy problems for two cases of the first order (see also [8] and [10, Theorem 3.3], where the abstract Cauchy problem was systematically studied).

To simplify our statements, we will consider the following abstract Cauchy problem in Sections 3 and 4 :

(ACP, T]

$$
\left\{\begin{array}{l}
u^{\prime}(t)=A u(t), \quad t \in[0, T], \\
u(0)=x,
\end{array}\right.
$$

where $0<T<\infty$ is given. As regards (ACP, $T$ ) with $0<T \leq \infty$, we will make a remark at the end of Section 3.

The paper is organized as follows.

In Section 2, we introduce some basic concepts and properties for local regularized semigroups; the corresponding concepts and properties for the global case can be found in [8] and [10].

In Section 3, we concentrate on establishing several Hille-Yosida type theorems for local regularized semigroups. Since we mainly consider the local case generated by closed operators, a new approach, distinct from the usual ones, is necessary. In particular, to reach our target, we have to "split" the quasi- $C$-resolvent (Definition 3.1) of the generator into two parts, where Arendt's vector-valued version of Widder's theorem for the Laplace transform is applicable to the first part, and the second part vanishes eventually (Theorem 3.2). When the generating operator is densely defined, although the asymptotic $C$-resolvents introduced in [14] become efficient, in our approach (Theorem 3.4) we do not have to assume that the range of $C$ is dense in $X$, which plays a crucial role in [14].

In Section 4, we deal with local integrated semigroups. Since we are able to apply the approach of Section 3, the results (Theorem 4.2) produced in this section are different from the corresponding ones established in [14, §4].

In the last Section 5, we present several examples to illustrate the applications of the theorems established in Sections 3 and 4.

Throughout, $\mathbb{C}$ is the complex plane; $X$ is a complex Banach space; $B(X)$ is the algebra of all bounded linear operators on $X ; A$ is a closed linear operator on $X ; C \in B(X)$ is always assumed to be injective. For a linear operator $S, D(S)$ and $\operatorname{Im}(S)$ are the domain and image of $S$, respectively, while $\varrho(S)$ and $\sigma(S)$ are the resolvent set and spectrum of $S$, respectively. 
2. Basic concepts and properties. We start this section with the following lemma.

Lemma $2.1[1$, Corollary 1.2]. Let $M>0, \omega \geq 0$ and let $\gamma:(0, \infty) \rightarrow X$ be a function. Then the following are equivalent:

(i) $\gamma$ is infinitely differentiable and

$$
\left\|(\lambda-\omega)^{n+1} \gamma^{(n)}(\lambda) / n !\right\| \leq M \quad \forall \lambda>\omega, n \in \mathbb{N} \cup\{0\} .
$$

(ii) There exists a function $F:[0, \infty) \rightarrow X$ satisfying $F(0)=0$ and

$$
\limsup _{h \downarrow 0} h^{-1}\|F(t+h)-F(t)\| \leq M e^{\omega t} \quad \forall t \geq 0
$$

$$
\gamma(\lambda)=\lambda \int_{0}^{\infty} e^{-\lambda t} F(t) d t \quad \forall \lambda>\omega .
$$

Definition $2.2[8,10,15]$. Assume $A$ is closed, $C \in B(X)$ is injective and $k \in \mathbb{N} \cup\{0\}$. A local mild $k$-times integrated $C$-existence family for $A$ is a strongly continuous family $\{W(t)\}_{t \in[0, T]} \subseteq B(X)$ of operators such that for all $x \in X$ and $t \in[0, T], \int_{0}^{t} W(s) x d s \in D(A)$ and

$$
A \int_{0}^{t} W(s) x d s=W(t) x-\frac{t^{k}}{k !} C x .
$$

Definition $2.3[10,15]$. Let $A, C$ be as in Definition 2.2 and $k \in \mathbb{N}$. A strongly continuous family $\{W(t)\}_{t \in[0, T]} \subseteq B(X)$ of operators is called a local $k$-times integrated $C$-regularized semigroup if $W(0)=0$ and

$$
\begin{array}{r}
W(s) W(t) x=\frac{1}{(k-1) !}\left[\int_{s}^{s+t}-\int_{0}^{t}\right](s+t-r)^{k-1} W(r) C x d r \\
\forall x \in X, 0 \leq s, t, s+t \leq T .
\end{array}
$$

A local 0-times integrated $C$-regularized semigroup is defined to be a local $C$-regularized semigroup.

$W(\cdot)$ is said to be nondegenerate if $W(t) x=0$, for all $t \in[0, T]$, implies $x=0$. A closed operator $A$ is called a subgenerator of the nondegenerate local $k$-times integrated $C$-regularized semigroup $\{W(t)\}_{t \in[0, T]}$ if:

(a) $W(t) A \subseteq A W(t)$ for all $t \in[0, T]$.

(b) $\{W(t)\}_{t \in[0, T]}$ is a local mild $k$-times integrated $C$-existence family for $A$.

The basic properties of nondegenerate local $k$-times integrated $C$-regularized semigroups, appearing in the following proposition, are similar to the well known properties of $C$-regularized semigroups (see [8], [10]).

Throughout, we will only consider the nondegenerate case and omit the term nondegenerate for simplicity. 
Proposition 2.4. Let $k \in \mathbb{N}$ and let $A$ be a subgenerator of the local $k$-times integrated $C$-regularized semigroup $\{W(t)\}_{t \in[0, T]}$. Then:

(i) $C A \subseteq A C$.

(ii) For $x \in X$ and $t \in[0, T]$, if $W(t) x$ is differentiable then $W(t) x \in$ $D(A)$ and

$$
A W(t) x=\frac{d}{d t}[W(t) x]-\frac{t^{k-1}}{(k-1) !} C x .
$$

(iii) If $x \in D(A)$ then $W(t) x$ is continuously differentiable on $[0, T]$ and

$$
W(t) A x=\frac{d}{d t}[W(t) x]-\frac{t^{k-1}}{(k-1) !} C x .
$$

(iv) All solutions of (ACP, T] are unique.

(v) $\{W(t)\}_{t \in[0, T]}$ is uniquely determined by one of its subgenerators.

(vi) Assume that $\{W(t)\}_{t \in[0, T]}$ is Lipschitz continuous and $A$ is densely defined. Then $W(t) x$ is continuously differentiable in $t \in[0, T]$ for every $x \in X$. Define $W_{0}(t) x:=(d / d t) W(t) x$ for every $x \in X$. Then $\left\{W_{0}(t)\right\}_{t \in[0, T]}$ is a local $(k-1)$-times $C$-regularized semigroup having $A$ as a subgenerator.

Proof. (i) follows from $W(t) A \subseteq A W(t)$ and

$$
\begin{aligned}
& A \int_{0}^{t} W(s) A x d s=W(t) A x-\frac{t^{k}}{k !} C A x, \\
& A \int_{0}^{t} W(s) A x d s=A\left[A \int_{0}^{t} W(s) x d s\right]=A W(t) x-\frac{t^{k}}{k !} A C x \quad \forall x \in D(A) .
\end{aligned}
$$

(ii) Differentiating both sides of

$$
A \int_{0}^{t} W(s) x d s=W(t) x-\frac{t^{k}}{k !} C x
$$

in $t$ yields (2.2).

(iii) follows from

$$
\int_{0}^{t} W(s) A x d s=A \int_{0}^{t} W(s) x d s=W(t) x-\frac{t^{k}}{k !} C x \quad \forall x \in D(A) .
$$

(iv) is similar to [8, Theorem 3.5].

(v) is similar to [10, Proposition 2.9].

(vi) Let $X_{0}$ be the set of all $x \in X$ so that $W(t) x$ is continuously differentiable on $[0, T]$. As in $[1$, p. 340] or [15, Lemma 5.1], we can show that $X_{0}$ is a closed subspace of $X$. Since $D(A) \subset X_{0}$ from (iii), we have $X_{0}=X$ when $A$ is densely defined. This gives (vi). 
The following lemma, which plays a crucial role in this paper, states that a strongly continuous family of bounded operators defined on $[0, T]$ is automatically a local $k$-times integrated semigroup if it satisfies (a) and (b) of Definition 2.3. The global case can be found in [10, Theorem 3.3], [15, Theorem 2.4] and [16, Theorem 2.4].

Lemma 2.5. Let $A$ be closed, $k \in \mathbb{N} \cup\{0\}$ and let $\{W(t)\}_{t \in[0, T]} \subseteq B(X)$ be a strongly continuous family of operators. Then $\{W(t)\}_{t \in[0, T]}$ is a local $k$-times integrated $C$-regularized semigroup having $A$ as a subgenerator if and only if:

(i) $W(t) A \subseteq A W(t)$ for all $t \in[0, T]$.

(ii) $\{W(t)\}_{t \in[0, T]}$ is a local mild $k$-times integrated $C$-existence family for $A$.

\section{Hille-Yosida type theorems for local once integrated $C$-regu-} larized semigroups. In this section we present several Hille-Yosida type theorems for local $C$-regularized semigroups and local once integrated $C$ regularized semigroups, and consider the local abstract Cauchy problems.

Definition 3.1. Assume $A$ is closed, $C \in B(X)$ is injective and $\omega \in \mathbb{R}$. A family $\{R(\lambda)\}_{\lambda \in(\omega, \infty)}$ is called a quasi-C-resolvent of $A$ if:

(a) $R(\lambda) x$ is infinitely differentiable in $\lambda>\omega$ for all $x \in X$.

(b) $R(\lambda) A \subseteq A R(\lambda), R(\lambda) R(\mu)=R(\mu) R(\lambda)$ for all $\lambda, \mu>\omega$.

(c) $R(\lambda) x \in D(A)$ and

$$
(\lambda-A) R(\lambda) x=C x+\lambda U(\lambda) x \quad \forall x \in X, \lambda>\omega,
$$

where $U(\lambda) x$ is infinitely differentiable in $\lambda>\omega$ for all $x \in X$ and there exists $M>0$ such that

$$
\begin{aligned}
&\left\|\left(\frac{d}{d \lambda}\right)^{n-1} U(\lambda) x\right\| \leq M T^{n-1} e^{-\lambda T}\|x\| \\
& \forall x \in X, \lambda>\max \{\omega, n / T\}, n \in \mathbb{N} .
\end{aligned}
$$

A quasi- $C$-resolvent of $A$ will be simply written as $R(\cdot)$ in some cases.

Theorem 3.2. Assume $A$ is closed and $C \in B(X)$ is injective. Then $A$ is a subgenerator of a local once integrated $C$-regularized semigroup $\{W(t)\}_{t \in[0, T]}$ that is Lipschitz continuous if and only if:

(i) There exists a quasi-C-resolvent $\{R(\lambda)\}_{\lambda>0}$ of $A$.

(ii) $R(\lambda)$ is the sum of two $B(X)$-valued functions $L(\lambda)$ and $V(\lambda)$ such that $L(\lambda) x$ and $V(\lambda) x$ are infinitely differentiable in $\lambda>0$ for all $x \in X$ and there exists $M>0$ such that

$$
\left\|\frac{\lambda^{n}}{(n-1) !}\left(\frac{d}{d \lambda}\right)^{n-1} L(\lambda) x\right\| \leq M\|x\| \quad \forall x \in X, \lambda>0, n \in \mathbb{N},
$$




$$
\left\|\left(\frac{d}{d \lambda}\right)^{n-1} V(\lambda) x\right\| \leq M T^{n-1} e^{-\lambda T}\|x\| \quad \forall x \in X, \lambda>n / T, n \in \mathbb{N} .
$$

Here we assume that $M$ is the same constant as in Definition 3.1.

Proof. Necessity. It is evident that the function

$$
F(t):= \begin{cases}W(t), & t \in[0, T], \\ W(T), & t \in(T, \infty),\end{cases}
$$

is Lipschitz continuous on $[0, \infty)$. Thus the function

$$
L(\lambda) x:=\lambda \int_{0}^{\infty} e^{-\lambda s} F(s) x d s \quad \forall \lambda>0
$$

is infinitely differentiable and satisfies (3.1) by Lemma 2.1. Define

$$
\begin{aligned}
R(\lambda) x & :=\lambda \int_{0}^{T} e^{-\lambda s} F(s) x d s \quad \forall x \in X, \lambda>0, \\
V(\lambda) & :=-e^{-\lambda T} F(T) \quad \forall \lambda>0 .
\end{aligned}
$$

Then $R(\lambda)=L(\lambda)+V(\lambda)$ and $V(\lambda) x$ is infinitely differentiable in $\lambda>0$ for all $x \in X$. Since

$$
\left|\left(\frac{d}{d \lambda}\right)^{n-1} e^{-\lambda T}\right|=T^{n-1} e^{-\lambda T} \quad \forall \lambda>0,
$$

$V(\cdot)$ satisfies (3.2). Moreover, from the definition of $F(\cdot)$ and $R(\cdot)$, we have $R(\lambda) A \subseteq A R(\lambda)$ for all $\lambda>0$.

We now show that $R(\cdot)$ is a quasi-resolvent of $A$. (a) and (b) of Definition 3.1 are clearly true with $\omega=0$. To prove (c), we use integration by parts to find

$$
\begin{aligned}
(\lambda-A) R(\lambda) x= & (\lambda-A) \lambda \int_{0}^{T} e^{-\lambda s} W(s) x d s \\
= & \lambda^{2} \int_{0}^{T} e^{-\lambda s} W(s) x d s-\lambda\left[A \int_{0}^{T} e^{-\lambda s} d \int_{0}^{s} W(r) x d r\right] \\
= & \lambda^{2} \int_{0}^{T} e^{-\lambda s} W(s) x d s-\lambda e^{-\lambda T}[W(T) x-T C x] \\
& -\lambda^{2} \int_{0}^{T} e^{-\lambda s}[W(s) x-s C x] d s \\
= & \left(1-e^{-\lambda T}\right) C x-\lambda e^{-\lambda T} W(T) x=C x+\lambda U(\lambda) x,
\end{aligned}
$$

where

$$
U(\lambda):=-\lambda^{-1} e^{-\lambda T} C-e^{-\lambda T} W(T) .
$$


For $\lambda \geq n / T$, we have

$$
\begin{aligned}
\left|\left(\frac{d}{d \lambda}\right)^{n-1}\left[\lambda^{-1} e^{-\lambda T}\right]\right| & =\left|\sum_{k=0}^{n-1} \frac{(n-1) !}{k !} \lambda^{-(n-k)} T^{k} e^{-\lambda T}\right| \\
& \leq n \lambda^{-1} T^{n-1} e^{-\lambda T} \leq T^{n} e^{-\lambda T}
\end{aligned}
$$

Here we used the fact that the terms in $\sum_{k=0}^{n-1}$ are increasing for given $\lambda \geq n / T$ as $k$ increases from 0 to $n-1$. Hence the value of $\sum_{k=0}^{n-1}$ is no more than $n$ times the last term. Thus (3.4) is true. From (3.3) and (3.4), $U(\cdot)$ satisfies the inequality in (c) of Definition 3.1 and $R(\cdot)$ is therefore a quasi- $C$-resolvent of $A$.

Sufficiency. From (3.1) and Lemma 2.1 there exists $F:[0, \infty) \rightarrow B(X)$ such that

$$
\begin{gathered}
F(0)=0, \quad\|F(s+h)-F(s)\| \leq M h \quad \forall s, h \geq 0, \\
L(\lambda) x=\lambda \int_{0}^{\infty} e^{-\lambda s} F(s) x d s \quad \forall \lambda>0 .
\end{gathered}
$$

From (ii) of the present theorem and (c) of Definition 3.1, we have

$$
\begin{aligned}
A\left[\frac{R(\lambda) x}{\lambda}\right] & =A\left[\int_{0}^{\infty} e^{-\lambda s} F(s) x d s+\lambda^{-1} V(\lambda) x\right] \\
A\left[\frac{R(\lambda) x}{\lambda}\right] & =R(\lambda) x-\lambda^{-1} C x-U(\lambda) x \\
& =\lambda \int_{0}^{\infty} e^{-\lambda s} F(s) x d s-\lambda^{-1} C x-[U(\lambda)-V(\lambda)] x,
\end{aligned}
$$

respectively. Therefore

$$
\begin{aligned}
A\left[\int_{0}^{\infty} e^{-\lambda s} F(s) x\right. & \left.d s+\lambda^{-1} V(\lambda) x\right] \\
& =\lambda \int_{0}^{\infty} e^{-\lambda s} F(s) x d s-\lambda^{-1} C x-[U(\lambda)-V(\lambda)] x .
\end{aligned}
$$

Since $A$ is closed, we can differentiate both sides $n-1$ times in $\lambda$ and multiply the resulting relation by $(-1)^{n-1} \lambda^{n} /(n-1)$ ! to find

$$
\begin{aligned}
A \frac{\lambda^{n}}{(n-1) !}\left[\int_{0}^{\infty} e^{-\lambda s} s^{n-1} F(s) x d s\right. & \\
& \left.+\sum_{k=0}^{n-1}(-1)^{k} \frac{(n-1) !}{k !} \lambda^{-(n-k)} V^{(k)}(\lambda) x\right]
\end{aligned}
$$




$$
\begin{aligned}
= & (-1)^{n-1} \frac{\lambda^{n}}{(n-1) !}\left(\frac{d}{d \lambda}\right)^{n-1}\left[\lambda \int_{0}^{\infty} e^{-\lambda s} F(s) x d s\right] \\
& +(-1)^{n} \frac{\lambda^{n}}{(n-1) !}\left[U^{(n-1)}(\lambda)-V^{(n-1)}(\lambda)\right] x-C x .
\end{aligned}
$$

For given $t \in(0, T)$, let $v \in(0, t]$ and set $\lambda=n / v$, then define

$$
\begin{aligned}
I_{0, n}(v) & :=\sum_{k=0}^{n-1}(-1)^{k} \frac{1}{k !}\left(\frac{n}{v}\right)^{k} V^{(k)}\left(\frac{n}{v}\right), \\
I_{1, n}(v) x & :=\frac{1}{(n-1) !}\left(\frac{n}{v}\right)^{n} \int_{0}^{\infty} e^{-(n / v) s} s^{n-1} F(s) x d s \\
& =\frac{n^{n}}{(n-1) !} \int_{0}^{\infty} e^{-n \mu} \mu^{n-1} F(v \mu) x d \mu \quad(s:=v \mu), \\
I_{2, n}(v) x & \left.:=\frac{(-1)^{n}}{(n-1) !}\left(\frac{n}{v}\right)^{n}\left(\frac{d}{d \lambda}\right)^{n-1}\left[\lambda \int_{0}^{\infty} e^{-\lambda s} F(s) x d s\right]\right]_{\lambda=n / v}, \\
I_{3, n}(v) x & :=\frac{(-1)^{n}}{(n-1) !}\left(\frac{n}{v}\right)^{n}\left[U^{(n-1)}\left(\frac{n}{v}\right)^{\left.-V^{(n-1)}\left(\frac{n}{v}\right)\right]_{x}},\right.
\end{aligned}
$$

and define $I_{j, n}(0)=0$ for $j=0,1,2,3$. From (3.2),

$$
\left\|I_{0, n}(v) x\right\| \leq M e^{-n T / v} \sum_{k=0}^{n-1} \frac{1}{k !}\left(\frac{n T}{v}\right)^{k}\|x\| \leq M \frac{n^{n}}{(n-1) !}\left(\frac{T}{v}\right)^{n-1} e^{-n T / v}\|x\|,
$$

which, combined with $I_{0, n}(0)=0$, approaches zero uniformly for $v \in[0, t]$ as $n \rightarrow \infty$. Here we note that the terms in $\sum_{k=0}^{n-1}$ are also increasing. Hence

$$
\lim _{n \rightarrow \infty} \int_{0}^{t} I_{0, n}(v) d v=0 .
$$

Given $0<\varepsilon<1$, with the help of the relations

$$
\begin{gathered}
\frac{n^{n}}{(n-1) !} \int_{0}^{\infty} e^{-n \mu} \mu^{n-1} d \mu=1, \\
\lim _{n \rightarrow \infty} \frac{n^{n}}{(n-1) !} \int_{0}^{1-\varepsilon} e^{-n \mu} \mu^{n-1} d \mu=0, \\
\lim _{n \rightarrow \infty} \frac{n^{n}}{(n-1) !} \int_{1+\varepsilon}^{\infty} e^{-n \mu} \mu^{n-1} d \mu=0,
\end{gathered}
$$

and the method applied in [12, p. 34], we find 


$$
\begin{aligned}
\lim _{n \rightarrow \infty}\left[I_{1, n}(v)-\right. & F(v)] x \\
& =\lim _{n \rightarrow \infty} \frac{n^{n}}{(n-1) !} \int_{0}^{\infty} e^{-n \mu} \mu^{n-1}[F(v \mu)-F(v)] x d \mu=0,
\end{aligned}
$$

uniformly for $v \in[0, t]$. Here we recall that $t \in(0, T)$ and $I_{1, n}(0)=F(0)=0$. Therefore

$$
\lim _{n \rightarrow 0} \int_{0}^{t} I_{1, n}(v) x d v=\int_{0}^{t} F(v) x d v
$$

To deal with $I_{2, n}(v) x$, let $x^{*} \in X^{*}$. Since $F(\cdot)$ is Lipschitz continuous on the interval $[0, \infty),\left\langle F(s) x, x^{*}\right\rangle$ is differentiable almost everywhere. Define $f_{x, x^{*}}(s):=(d / d s)\left\langle F(s) x, x^{*}\right\rangle$. Using integration by parts, we have

$$
\begin{aligned}
\int_{0}^{t}\left\langle I_{2, n}(v) x, x^{*}\right\rangle d v & =\frac{n^{n}}{(n-1) !} \int_{0}^{t} \int_{0}^{\infty} e^{-(n / v) s} v^{-n} s^{n-1} f_{x, x^{*}}(s) d s d v \\
& =\frac{n^{n}}{(n-1) !} \int_{0}^{t} \int_{0}^{\infty} e^{-n \mu} \mu^{n-1} f_{x, x^{*}}(v \mu) d \mu d v \quad(s:=v \mu) .
\end{aligned}
$$

By an argument similar to one used in the proof of (3.8), we can show that

$$
\lim _{n \rightarrow \infty} \int_{0}^{t}\left\langle I_{2, n}(v) x, x^{*}\right\rangle d v=\int_{0}^{t} f_{x, x^{*}}(v) d v=\left\langle F(t) x, x^{*}\right\rangle .
$$

As regards $I_{3, n}(v) x$, we have

$$
\begin{aligned}
\left\|I_{3, n}(v) x\right\| & \leq \frac{1}{(n-1) !}\left(\frac{n}{v}\right)^{n}\left[\left\|U^{(n-1)}\left(\frac{n}{v}\right)\right\|+\left\|V^{(n-1)}\left(\frac{n}{v}\right)\right\|\right]\|x\| \\
& \leq \frac{2 M}{T} \cdot \frac{n^{n}}{(n-1) !}\left(\frac{T}{v}\right)^{n} e^{-n(T / v)}\|x\| \rightarrow 0 \quad \text { as } n \rightarrow \infty,
\end{aligned}
$$

uniformly for $v \in[0, t]$. Here we also recall that $t \in(0, T)$ and $I_{3, n}(0)=0$. Therefore

$$
\lim _{n \rightarrow \infty} \int_{0}^{t} I_{3, n}(v) x d v=0 .
$$

Combining (3.7)-(3.10) shows that, for $t \in[0, T)$,

$$
A \int_{0}^{t} F(s) x d s=F(t) x-t C x \quad \forall x \in X .
$$

Since $F(\cdot)$ is continuous on $[0, T]$ and $A$ is closed, (3.11) remains true for $t \in[0, T]$. 
We now show that for every $x \in D(A), F(t) x \in D(A)$ and $A F(t) x=$ $F(t) A x$. From the hypotheses in (ii) we have

$$
\frac{R(\lambda) A x}{\lambda}=\int_{0}^{\infty} e^{-\lambda s} F(s) A x d s+\lambda^{-1} V(\lambda) A x .
$$

Since $R(\lambda) A x=A R(\lambda) x$, instead of (3.5), we have

$$
\begin{aligned}
\int_{0}^{\infty} e^{-\lambda s} F(s) A x d s+ & \lambda^{-1} V(\lambda) A x \\
& =\lambda \int_{0}^{\infty} e^{-\lambda s} F(s) x d s-\lambda^{-1} C x-[U(\lambda)-V(\lambda)] x .
\end{aligned}
$$

Hence, instead of (3.6), we have

$$
\begin{aligned}
\frac{\lambda^{n}}{(n-1) !}\left[\int_{0}^{\infty} e^{-\lambda s} F(s) A\right. & \left.x d s+\sum_{k=0}^{n-1}(-1)^{k} \frac{(n-1) !}{k !} \lambda^{-(n-k)} V^{(k)}(\lambda) A x\right] \\
= & (-1)^{n} \frac{\lambda^{n}}{(n-1) !}\left(\frac{d}{d \lambda}\right)^{n-1}\left[\lambda \int_{0}^{\infty} e^{-\lambda s} F(s) x d s\right] \\
& +(-1)^{n} \frac{\lambda^{n}}{(n-1) !}\left[U^{(n-1)}(\lambda)-V^{(n-1)}(\lambda)\right] x-C x
\end{aligned}
$$

Then

$$
I_{1, n}(v) A x=\frac{n^{n}}{(n-1) !} \int_{0}^{\infty} e^{-n \mu} \mu^{n-1} F(v \mu) A x d \mu,
$$

and, instead of (3.8), the following holds:

$$
\lim _{n \rightarrow \infty} \int_{0}^{t} I_{1, n}(v) A x d v=\int_{0}^{t} F(v) A x d v
$$

This, combined with (3.7), (3.9) and (3.10) where all $x$ 's are assumed to be in $D(A)$, gives rise to

$$
\int_{0}^{t} F(s) A x d s=F(t) x-C x \quad \forall x \in D(A), t \in[0, T] .
$$

From (3.11) and (3.12),

$$
A \int_{0}^{t} F(s) x d s=\int_{0}^{t} F(s) A x d s
$$

$A$ being closed, we can differentiate both sides in $t$ to find $A F(t) x=F(t) A x$. Set $W(t):=F(t)$. From Lemma $2.5,\{W(t)\}_{t \in[0, T]}$ is a local once integrated $C$-regularized semigroup having $A$ as a subgenerator. 
It is worthwhile to mention that, instead of (3.9), we can show that the following is true in the strong operator topology:

$$
\lim _{n \rightarrow \infty} \int_{0}^{t} I_{2, n}(v) x d v=F(t) x \quad \forall x \in X .
$$

The details are omitted.

The following definition can be found in [14, Definition 2].

Definition 3.3. Assume $A$ is closed, $C \in B(X)$ is injective and $\omega \in \mathbb{R}$. A family $\{L(\lambda)\}_{\lambda>\omega} \subseteq B(X)$ is called an asymptotic $C$-resolvent of $A$ if:

(a) $L(\lambda) x$ is infinitely differentiable in $\lambda>\omega$ for all $x \in X$.

(b) $L(\lambda) A \subseteq A L(\lambda), L(\lambda) L(\mu)=L(\mu) L(\lambda)$ for all $\lambda, \mu>\omega$.

(c) $L(\lambda) x \in D(A)$ and

$$
(\lambda-A) L(\lambda) x=C x+\widetilde{U}(\lambda) x \quad \forall x \in X, \lambda>\omega,
$$

where $\widetilde{U}(\lambda) x$ is infinitely differentiable in $\lambda>\omega$ for all $x \in X$ and there exists $M>0$ such that

$$
\begin{aligned}
&\left\|\left(\frac{d}{d \lambda}\right)^{n-1} \widetilde{U}(\lambda) x\right\| \leq M T^{n-1} e^{-\lambda T}\|x\| \\
& \forall x \in X, \lambda>\max \{\omega, n / T\}, n \in \mathbb{N} .
\end{aligned}
$$

The following theorem deals with the case where $A$ is closed and densely defined. As indicated in Section 1, in our approach, it is not necessary to assume that $\operatorname{Im}(C)$ is dense in $X$. This is more general than the Hille-Yosida type theorem appearing in [14, Theorem 2.1].

Theorem 3.4. Assume that $A$ is closed and densely defined, and $C \in$ $B(X)$ is injective. Then $A$ is a subgenerator of a local $C$-regularized semigroup $\left\{W_{0}(t)\right\}_{t \in[0, T]}$ if and only if there exists an asymptotic $C$-resolvent $\{L(\lambda)\}_{\lambda>0}$ of A satisfying (3.1) of Theorem 3.2.

Proof. Necessity. Let $\left\{W_{0}(t)\right\}_{t \in[0, T]}$ be a local $C$-regularized semigroup having $A$ as a subgenerator. Define

$$
L(\lambda) x:=\int_{0}^{T} e^{-\lambda s} W_{0}(s) x d s \quad \forall x \in X, \lambda>0 .
$$

By the argument applied in [14, Proposition 2.1], we can show that (3.1) is true and $L(\cdot)$ is an asymptotic $C$-resolvent of $A$.

Sufficiency. Let $F:[0, \infty) \rightarrow B(X)$ satisfy

$$
\begin{aligned}
& F(0)=0, \quad\|F(s+h)-F(s)\| \leq M h \quad \forall s, h>0, \\
& L(\lambda) x:=\lambda \int_{0}^{\infty} e^{-\lambda s} F(s) x d s .
\end{aligned}
$$


From Definition 3.3,

$$
A \int_{0}^{\infty} e^{-\lambda s} F(s) x d s=\lambda \int_{0}^{\infty} e^{-\lambda s} F(s) x d s-\lambda^{-1} C x-\lambda^{-1} \widetilde{U}(\lambda) x \quad \forall \lambda>0 .
$$

Repeating the argument of Theorem 3.2 we find that $\int_{0}^{t} F(s) x d s \in D(A)$ and

$$
A \int_{0}^{t} F(s) x d s=F(t) x-t C x \quad \forall x \in X, t \in[0, T],
$$

and that $F(t) A \subseteq A F(t)$. Set $W(t):=F(t)$ for $t \in[0, T]$. Then $\{W(t)\}_{t \in[0, T]}$ is a local once integrated $C$-regularized semigroup having $A$ as a subgenerator. Since $A$ is densely defined, from Proposition 2.4(vi), $W_{0}(t) x:=$ $(d / d t) W(t) x$ is a local $C$-regularized semigroup having $A$ as a subgenerator.

REMARKS 3.5. (i) We now show that the asymptotic $C$-resolvent appearing in (3.13) is consistent with that in (3.14). In fact, integrate the right-hand side of (3.13) by parts to find

$$
L(\lambda) x=\lambda \int_{0}^{T} e^{-\lambda t} \int_{0}^{t} W_{0}(s) x d s d t+e^{-\lambda T} \int_{0}^{T} W_{0}(s) x d s .
$$

If we define

$$
F(t) x:= \begin{cases}\int_{0}^{t} W_{0}(s) x d s, & t \in[0, T] \\ \int_{0}^{T} W_{0}(s) x d s, & t \in(T, \infty)\end{cases}
$$

then

$$
L(\lambda) x=\lambda \int_{0}^{\infty} e^{-\lambda t} F(t) x d t
$$

which is given in (3.14).

(ii) It is also clear that in Theorem 3.2 , if $A$ is densely defined, the operator $L(\lambda)$ defined there coincides with that in Theorem 3.4, hence $L(\lambda)$, in both Theorems 3.2 and 3.4, is different from $R(\lambda)$ in Theorem 3.2, that is, a quasi- $C$-resolvent is different from an asymptotic $C$-resolvent.

(iii) As regards the local once integrated $C$-regularized semigroups defined on $[0, T)$, instead of Theorem 3.2 , we have

TheOREM 3.6. $A$ is a subgenerator of a local once integrated $C$-regularized semigroup $\{W(t)\}_{t \in[0, T)}$ that is locally Lipschitz continuous if and only if:

(i) For every $\tau \in(0, T)$ there exists a quasi-C-resolvent $\left\{R_{\tau}(\lambda)\right\}_{\lambda>0}$ of $A$. 
(ii) $R_{\tau}(\lambda)$ is the sum of two $B(X)$-valued functions $L_{\tau}(\lambda)$ and $V_{\tau}(\lambda)$ such that $L_{\tau}(\lambda) x$ and $V_{\tau}(\lambda) x$ are infinitely differentiable in $\lambda>0$ for all $x \in X$ and there exists $M_{\tau}>0$ such that $L_{\tau}(\lambda)$ and $V_{\tau}(\lambda)$ satisfy (3.1) and (3.2) with $M$ replaced by $M_{\tau}$ and $T$ in (3.2) replaced by $\tau$, respectively.

Proof. Necessity. Instead of the operator-valued functions defined in the proof of Theorem 3.2, we now define, for given $\tau \in(0, T)$,

$$
F_{\tau}(t):= \begin{cases}W(t), & t \in[0, \tau] \\ W(\tau), & t \in(\tau, \infty)\end{cases}
$$

and, for all $\lambda>0$,

$$
\begin{array}{lll}
L_{\tau}(\lambda) x:=\lambda \int_{0}^{\infty} e^{-\lambda s} F_{\tau}(s) x d s, & V_{\tau}(\lambda):=-e^{-\lambda \tau} F_{\tau}(\tau), \\
R_{\tau}(\lambda) x:=\lambda \int_{0}^{\tau} F_{\tau}(s) x d s, & U_{\tau}(\lambda):=-\frac{1}{\lambda} e^{-\lambda \tau} C-e^{-\lambda \tau} W(\tau) .
\end{array}
$$

Using the proof of the necessity of Theorem 3.2 we can show that (i) and (ii) of the present theorem are true.

Sufficiency. For given $\tau \in(0, T)$, from Lemma 2.1 there exists $F_{\tau}$ : $[0, \infty) \rightarrow B(X)$ such that

$$
F_{\tau}(0)=0, \quad\left\|F_{\tau}(s+h)-F_{\tau}(s)\right\| \leq M_{\tau} h \quad \forall s, h>0 .
$$

Using the proof of the sufficiency of Theorem 3.2, we can show that $\left\{F_{\tau}(t)\right\}_{t \in[0, \tau]}$ is a local once integrated $C$-regularized semigroup having $A$ as a subgenerator by Lemma 2.5.

From Proposition 2.4(v), for $0<\tau<\tau^{\prime}<T$, we have $F_{\tau}(t)=F_{\tau^{\prime}}(t)$ whenever $0 \leq t \leq \min \left\{\tau, \tau^{\prime}\right\}$, that is, $F_{\tau}(t)$ is independent of $\tau$ whenever $t \in[0, \tau]$. This enables us to define

$$
W(t):=F_{\tau}(t) \quad \forall t \in[0, T),
$$

where $\tau$ is chosen so that $t \leq \tau<T$. Then $\{W(t)\}_{t \in[0, T)}$ is a local once integrated $C$-regularized semigroup having $A$ as a subgenerator.

In the following we deal with the local abstract Cauchy problems (ACP, T] and (ACP, $T$ ) with $0<T<\infty$ and $0<T \leq \infty$, respectively.

Definition 3.7 [5]. A solution of (ACP, T] is defined to be a function $u(\cdot, x) \in C^{1}([0, T], X)$ satisfying $(\mathrm{ACP}, T]$.

A mild solution of $(\mathrm{ACP}, T]$ is a function $u(\cdot, x) \in C([0, T], X)$ such that $v(t, x):=\int_{0}^{t} u(s, x) d s \in D(A)$ and 


$$
v^{\prime}(t, x)=A v(t, x)+x \quad \forall t \in[0, T] .
$$

The definitions of solutions and mild solutions of $(\mathrm{ACP}, T)$ are similar.

Theorem 3.8. Assume $A$ is closed, $C \in B(X)$ is injective and $C A \subseteq$ $A C$. Then the following are equivalent:

(i) $A$ is a subgenerator of a local $C$-regularized semigroup on $[0, T]$ (resp. on $[0, T)$ ).

(ii) $(\mathrm{ACP}, T]($ resp. $(\mathrm{ACP}, T))$ has a unique mild solution for all $x \in$ $\operatorname{Im}(C)$.

(iii) All solutions of $(\mathrm{ACP}, T]$ (resp. $(\mathrm{ACP}, T))$ are unique and there is a local mild $C$-existence family $\{W(t)\}_{t \in[0, T]}$ (resp. $\left.\{W(t)\}_{t \in[0, T)}\right)$ for A.

(iv) There exists a local mild $C$-existence family $\{W(t)\}_{t \in[0, T]}$ (resp. $\left.\{W(t)\}_{t \in[0, T)}\right)$ for $A$ such that $W(t) A \subseteq A W(t)$ for all $t \in[0, T]$ (resp. $t \in[0, T))$.

If , in addition, $\varrho(A)$ is nonempty, then (i)-(iv) are equivalent to

(v) $(\mathrm{ACP}, T]$ (resp. $(\mathrm{ACP}, T))$ has a unique solution for all $x \in C(D(A))$.

The proof of this theorem is almost the same as those of $[8$, Theorem 4.15] and [10, Theorem 3.3].

4. Local integrated semigroups. In this section we deal with local integrated semigroups.

Definition 4.1 [14]. Let $k \in \mathbb{N} \cup\{0\}, 0<T<\infty$. A strongly continuous family $\{S(t)\}_{t \in[0, T]} \subseteq B(X)$ of operators is a local $k$-times integrated semigroup if it is a local $k$-times integrated $I$-regularized semigroup (see Definition 2.3).

The generator $A$ of a nondegenerate $\{S(t)\}_{t \in[0, T]}$ is defined by

$$
\begin{aligned}
& D(A):=\{x \in X \mid \text { there exists } y \in X \text { such that } \\
& \left.\qquad \int_{0}^{t} S(s) y d s=S(t) x-\frac{t^{k}}{k !} x \forall t \in[0, T]\right\},
\end{aligned}
$$

$A x:=y$.

It is easily seen that $y$, if it exists, is uniquely determined by $x$ in view of the nondegeneracy of $\{S(t)\}_{t \in[0, T]}$.

In the following we will only deal with nondegenerate local $k$-times integrated semigroups. From Definition 2.3, a local integrated semigroup may also have subgenerators. But from [2, Proposition 3.1(d)], all subgenerators are identical. Therefore in the theory of local integrated semigroups, only generators have to be considered. 
From [2, Theorems 2.1, 2.2 and Proposition 2.3], there exists $k \in \mathbb{N} \cup\{0\}$ such that $A$ generates a local $k$-times integrated semigroup if and only if:

(i) $\varrho(A)$ is nonempty and $E(\alpha, \beta) \subseteq \varrho(A)$ for some $\alpha, \beta>0$, where

$$
E(\alpha, \beta):=\left\{\lambda \in \mathbb{C}|\operatorname{Re} \lambda \geq \beta,| \operatorname{Im} \lambda \mid \leq e^{\alpha \operatorname{Re} \lambda}\right\} .
$$

(ii) there exist $l \in \mathbb{N}$ and $M>0$ such that

$$
\left\|(\lambda-A)^{-1}\right\| \leq M(1+|\lambda|)^{l} \quad \forall \lambda \in E(\alpha, \beta) .
$$

When $A$ generates a local $k$-times integrated semigroup, $l$ in (ii) may be chosen equal to $k$. Conversely, if (i) and (ii) are true, $k$ should be greater than $l+1$. Moreover, when $A$ generates a local $k$-times integrated semigroup, the domains of solutions of the local abstract Cauchy problem are different from the domains of solutions when (i) and (ii) are true. Therefore $[2$, Theorems 2.1, 2.2 and Proposition 2.3] are far from an equivalence. Thus it is still necessary to establish a Hille-Yosida type theorem for local integrated semigroups. Such a theorem was given in [14, Theorem 4.7] under the assumption that the generators were densely defined. In the following, we will present another Hille-Yosida type theorem without assuming the generators to be densely defined. We can also see that, by using Lemma 2.5, the proof of the equivalence of (i) and (iii) of the following theorem is straightforward.

Theorem 4.2. Assume that $A$ is closed and $k \in \mathbb{N} \cup\{0\}$. Then the following are equivalent:

(i) $A$ is the generator of a local $(k+1)$-times integrated semigroup $\{S(t)\}_{t \in[0, T]}$ that is Lipschitz continuous.

(ii) $\varrho(A)$ is nonempty and $A$ generates a local $R\left(\lambda_{0}, A\right)^{k+1}$-regularized semigroup $\{W(t)\}_{t \in[0, T]}$ that is Lipschitz continuous, where $\lambda_{0} \in \varrho(A)$.

(iii) There exist $\alpha, \beta>0$ such that $E(\alpha, \beta) \subseteq \varrho(A)$ and for $\lambda>\beta$, $R(\lambda, A) / \lambda^{k}$ is the sum of two $B(X)$-valued functions $L(\lambda)$ and $V(\lambda)$ such that for all $x \in X, L(\lambda) x$ and $V(\lambda) x$ are infinitely differentiable in $\lambda>\beta$. Moreover, there exists $M>0$ such that

$$
\left\|\frac{\lambda^{n}}{(n-1) !}\left(\frac{d}{d \lambda}\right)^{n-1} L(\lambda) x\right\| \leq M\|x\| \quad \forall x \in X, \lambda>\beta, n \in \mathbb{N} .
$$

For every $t_{0} \in(0, T)$, there exists $M_{t_{0}}>0$ such that

$$
\begin{aligned}
&\left\|\left(\frac{d}{d \lambda}\right)^{n-1} V(\lambda) x\right\| \leq M_{t_{0}} t_{0}^{n-1} e^{-\lambda t_{0}}\|x\| \\
& \forall x \in X, \lambda>\max \left\{\beta, n / t_{0}\right\}, n \in \mathbb{N} .
\end{aligned}
$$


When (i)-(iii) are true, we have

$$
\begin{aligned}
W(t) x & =\left(\frac{d}{d t}\right)^{k+1} S(t)\left(\lambda_{0}-A\right)^{k+1} x, \\
S(t) x & =\left(\lambda_{0}-A\right)^{k+1} \int_{0}^{t} \frac{(t-s)^{k}}{k !} W(s) x d s \quad \forall t \in[0, T], x \in X .
\end{aligned}
$$

Proof. (i) $\Rightarrow$ (ii). From [2, Theorem 2.1], $\varrho(A)$ is nonempty. Fix $\lambda \in \varrho(A)$. As in [8, Theorem 18.3], we define

$$
W(t) x:=\left(\frac{d}{d t}\right)^{k+1} S(t) R\left(\lambda_{0}, A\right)^{k+1} x \quad \forall x \in X, t \in[0, T] .
$$

By using (4.4) and

$$
\frac{d}{d t} S(t) R\left(\lambda_{0}, A\right)^{k+1} x=S(t) A R\left(\lambda_{0}, A\right)^{k+1} x+\frac{t^{k}}{k !} R\left(\lambda_{0}, A\right)^{k+1} x \quad \forall x \in X,
$$

an induction argument shows that

$$
W(t) x=S(t) A^{k+1} R\left(\lambda_{0}, A\right)^{k+1} x+\sum_{j=0}^{k} \frac{t^{j}}{j !} A^{j} R\left(\lambda_{0}, A\right)^{k+1} x .
$$

This implies that $\{W(t)\}_{t \in[0, T]}$ is Lipschitz continuous and $W(t) A \subseteq A W(t)$. From

$$
\begin{aligned}
A \int_{0}^{t} W(s) x d s= & A \int_{0}^{t} S(s) A^{k+1} R\left(\lambda_{0}, A\right)^{k+1} x d s+\sum_{j=1}^{k+1} \frac{t^{j}}{j !} A^{j} R\left(\lambda_{0}, A\right)^{k+1} x \\
= & S(t) A^{k+1} R\left(\lambda_{0}, A\right)^{k+1} x-\frac{t^{k}}{(k+1) !} A^{k+1} R\left(\lambda_{0}, A\right)^{k+1} x \\
& +\sum_{j=1}^{k+1} \frac{t^{j}}{j !} A^{j} R\left(\lambda_{0}, A\right)^{k+1} x \\
= & W(t) x-R\left(\lambda_{0}, A\right)^{k+1} x
\end{aligned}
$$

and Lemma 2.5, $\{W(t)\}_{t \in[0, T]}$ is a local $R\left(\lambda_{0}, A\right)^{k+1}$-regularized semigroup. From $\left(\lambda_{0}-A\right)^{k+1} A R\left(\lambda_{0}, A\right)^{k+1}=A$ and [10, Proposition 2.9], we conclude that $A$ is the generator of $\{W(t)\}_{t \in[0, T]}$.

(ii) $\Rightarrow($ i). Define (see [8, Theorem 18.3])

$$
S(t) x:=\left(\lambda_{0}-A\right)^{k+1} \int_{0}^{t} \frac{(t-s)^{k}}{k !} W(s) x d s .
$$

Integrate the right-hand side by parts to find 


$$
\begin{aligned}
S(t) x=\left(\lambda_{0}-A\right)^{k+1} \int_{0}^{t} \frac{(t-s)^{k-1}}{(k-1) !}\left[\int_{0}^{s} W(r) x d r\right] d s & \\
& \forall x \in X, t \in[0, T] .
\end{aligned}
$$

Hence

$$
\begin{aligned}
A \int_{0}^{t} S(s) x d s & =\left(\lambda_{0}-A\right)^{k+1} \int_{0}^{t} \frac{(t-s)^{k}}{k !}\left[W(s) x-R\left(\lambda_{0}, A\right)^{k+1} x\right] d s \\
& =S(t) x-\frac{t^{k+1}}{(k+1) !} x .
\end{aligned}
$$

Lemma 2.5 implies that $\{S(t)\}_{t \in[0, T]}$ is a local $(k+1)$-times integrated semigroup generated by $A$.

By translating $A$, we may assume that $\lambda_{0}=0$. (4.5) implies that

$$
\begin{aligned}
S(t) x & =(-1)^{k+1} A^{k+1} \int_{0}^{t} \frac{(t-s)^{k}}{k !} W(s) x d s \\
& =(-1)^{k+1} W(t) x-\sum_{j=0}^{k} \frac{t^{j}}{j !} A^{-(k+1-j)} x .
\end{aligned}
$$

Hence $\{S(t)\}_{t \in[0, T]}$ is Lipschitz continuous.

(i) $\Rightarrow$ (iii). Similarly to Theorem 3.2 , define

$$
\begin{aligned}
& F(t):= \begin{cases}S(t), & t \in[0, T], \\
S(T), & t \in(T, \infty) ;\end{cases} \\
& L(\lambda) x:=\lambda \int_{0}^{\infty} e^{-\lambda s} F(s) x d s \quad \forall \lambda>0 .
\end{aligned}
$$

Then $L(\cdot)$ satisfies $(4.2)$ and $L(\lambda) x=\lambda \int_{0}^{T} e^{-\lambda s} S(s) x d s+e^{-\lambda T} S(T) x$.

A direct calculation shows that

$$
\begin{aligned}
& (\lambda-A)\left[\lambda \int_{0}^{T} e^{-\lambda s} S(s) x d s\right] \\
& \quad=\left[\frac{1}{\lambda^{k}}-e^{-\lambda T} q(\lambda)-\lambda e^{-\lambda T} S(T)\right] x \quad \forall \lambda>0, x \in X,
\end{aligned}
$$

where $q(\lambda)=\sum_{j=0}^{k} T^{j} /\left(j ! \lambda^{k-j}\right)$. Let $\alpha^{\prime}>\alpha, \beta^{\prime}>\beta$ and $\omega_{0}>0, \eta_{0}>0$ be sufficiently large, and let $\Gamma=\Gamma_{1} \cup \Gamma_{2}$ be a contour with

$$
\begin{aligned}
& \Gamma_{1}=\left\{\zeta=\xi+i \eta\left|\xi=\alpha^{\prime} \log \right| \eta\left|+\beta^{\prime},\right| \eta|\geq| \eta_{0} \mid\right\}, \\
& \Gamma_{2}=\left\{\zeta=\omega_{0}+i \eta|| \eta \mid \leq \eta_{0}\right\}
\end{aligned}
$$

oriented from the lower to upper half plane (see the proof of [13, Main Theorem] for details). Now assume $\lambda>\omega_{0}$ and $t_{0} \in(0, T)$. From (4.6) we 
may write

$$
\begin{aligned}
\frac{R(\lambda, A) x}{\lambda^{k}}= & \lambda \int_{0}^{T} e^{-\lambda s} S(s) x d s+e^{-\lambda T} q(\lambda) R(\lambda, A) x+\lambda e^{-\lambda T} R(\lambda, A) S(T) x \\
= & L(\lambda) x-e^{-\lambda T} S(T) x \\
& +\frac{e^{-\lambda t_{0}}}{2 \pi i} \int_{\Gamma}(\lambda-z)^{-1} e^{-z\left(T-t_{0}\right)} q(z) R(z, A) x d z \\
& +\frac{e^{-\lambda t_{0}}}{2 \pi i} \int_{\Gamma} z(\lambda-z)^{-1} e^{-z\left(T-t_{0}\right)} R(z, A) S(\tau) x d z \\
& =L(\lambda) x+V(\lambda) x \quad \forall x \in X .
\end{aligned}
$$

where $V(\lambda) x$ is the sum of the last three terms. From the inequality $0<$ $t_{0}<T$ and (4.1), those two integrals in $V(\lambda)$ define bounded linear operators on $X$. Applying (3.3) to $e^{-\lambda T}$ and $e^{\lambda t_{0}}$, we conclude that $V(\lambda)$ satisfies $(4.3)$. Here we applied the following relation to the first term in $V(\lambda)$ :

$$
T^{n-1} e^{-\lambda T} \leq t_{0}^{n-1} e^{-\lambda t_{0}} \quad \forall \lambda>\max \left\{\beta, n / t_{0}\right\}
$$

$($ iii $) \Rightarrow($ i). From Lemma 2.1 there exists $F:[0, \infty) \rightarrow B(X)$ such that

$$
\begin{gathered}
F(0)=0, \quad \lim _{h \rightarrow 0} \frac{1}{h}\|F(s+h)-F(s)\| \leq M e^{\beta s} \quad \forall s \geq 0, \\
L(\lambda) x=\lambda \int_{0}^{\infty} e^{-\lambda s} F(s) x d s \quad \forall \lambda>\beta .
\end{gathered}
$$

The relation $R(\lambda, A) / \lambda^{k}=L(\lambda)+V(\lambda)$, together with the last equality, gives rise to

$$
\begin{aligned}
A\left[\frac{R(\lambda, A)}{\lambda^{k+1}} x\right] & =A\left[\int_{0}^{\infty} e^{-\lambda s} F(s) x d s+\lambda^{-1} V(\lambda) x\right], \\
A\left[\frac{R(\lambda, A)}{\lambda^{k+1}} x\right] & =\frac{R(\lambda, A)}{\lambda^{k}} x-\frac{1}{\lambda^{k+1}} x \\
& =\lambda \int_{0}^{\infty} e^{-\lambda s} F(s) x d s+V(\lambda) x-\frac{1}{\lambda^{k+1}} x .
\end{aligned}
$$

Therefore

$$
A\left[\int_{0}^{\infty} e^{-\lambda s} F(s) x d s+\lambda^{-1} V(\lambda) x\right]=\lambda \int_{0}^{\infty} e^{-\lambda s} F(s) x d s+V(\lambda) x-\frac{1}{\lambda^{k+1}} x .
$$

$A$ being closed, we can differentiate both sides $n-1$ times in $\lambda$ and multiply 
the resulting relation by $(-1)^{n-1} \lambda^{n} /(n-1)$ ! to find

$$
\begin{aligned}
A \frac{\lambda^{n}}{(n-1) !}[ & \int_{0}^{\infty} e^{-\lambda s} s^{n-1} F(s) x d s \\
& \left.+\sum_{j=0}^{n-1}(-1)^{j} \frac{(n-1) !}{j !} \lambda^{-(n-j)} V^{(j)}(\lambda) x\right] \\
= & (-1)^{n-1} \frac{\lambda^{n}}{(n-1) !}\left(\frac{d}{d \lambda}\right)^{n-1}\left[\lambda \int_{0}^{\infty} e^{-\lambda s} F(s) x d s\right] \\
& +(-1)^{n-1} \frac{\lambda^{n}}{(n-1) !} V^{(n-1)}(\lambda) x-\frac{(n+k-1) !}{k !(n-1) !} \lambda^{-k} x .
\end{aligned}
$$

For given $t \in(0, T)$, let $v \in(0, t]$ and set $\lambda=n / v$. Then integrate the last term in $v$ and take the limit to find

$$
\lim _{n \rightarrow \infty}\left[\frac{(n+k-1) !}{k !(n-1) ! n^{k}} \int_{0}^{t} v^{k} d v\right] x=\frac{t^{k+1}}{(k+1) !} x .
$$

Applying the same argument used in Theorem 3.2 to deal with all other terms in (4.7), we conclude that

$$
A \int_{0}^{t} F(s) x d s=F(t) x-\frac{t^{k+1}}{(k+1) !} x \quad \forall x \in X, t \in[0, T] .
$$

The fact that $F(\lambda) A \subseteq A F(\lambda)$ can be proved by the same method used in Theorem 3.2. Set $S(t):=F(t)$. From Lemma 2.5, $\{S(t)\}_{t \in[0, T]}$ is the $(k+1)$-times integrated semigroup generated by $A$.

Remarks 4.3. (i) If $T=\infty$ then Theorem 4.2 offers two equivalent conditions for a closed operator $A$ (not necessarily densely defined) generating a global $(k+1)$-times integrated semigroup which may not be exponentially bounded. Such an example can be found in [8] (see [8, Example 18.2] for details), therefore even if $T=\infty, V(\lambda)$ may not vanish automatically. In fact, if we set $t_{0} \rightarrow \infty$ in (4.3), it is very hard to conclude whether or not the factor $M_{t_{0}} t_{0}^{n-1} e^{-\lambda t_{0}}$ approaches zero.

(ii) If $V(\lambda)$ in Theorem 4.2 vanishes then the integrated semigroup generated by $A$ will be exponentially bounded and the equivalence (i) $\Leftrightarrow$ (iii) reduces to Arendt's theorem (see [1]).

(iii) As regards the local integrated semigroups defined on $[0, T)$, we also have a remark similar to Theorem 3.6. In order to save space, the details are omitted.

The following theorem states the relationship between the local Cauchy problem (ACP, $T$ ] (resp. (ACP, $T)$ ) and the closed operator $A$ generating 
a local $k$-times integrated semigroup. Its proof is the same as that of $[8$, Theorem 18.3].

Theorem 4.4. Assume $A$ is closed and $k \in \mathbb{N} \cup\{0\}$. Then the following are equivalent: $[0, T))$.

(i) A generates a local k-times integrated semigroup on $[0, T]$ (resp. on

(ii) (ACP, $T]$ (resp. $(\mathrm{ACP}, T))$ has a unique mild solution for all $x \in$ $D\left(A^{k}\right)$.

(iii) $\varrho(A)$ is nonempty and $(\mathrm{ACP}, T]$ (resp. $(\mathrm{ACP}, T))$ has a unique solution for all $x \in D\left(A^{k+1}\right)$.

(iv) $\varrho(A)$ is nonempty and $A$ generates a local $\left(\lambda_{0}-A\right)^{-k}$-regularized semigroup $\{W(t)\}_{t \in[0, T]}\left(\right.$ resp. $\left.\{W(t)\}_{t \in[0, T)}\right)$, where $\lambda_{0} \in \varrho(A)$.

5. Examples. We now present several examples.

EXAMPLE 5.1 [2]. (a) Let $\Sigma$ be a $\sigma$-finite measure space and let $1 \leq$ $p \leq \infty$. Assume $M: \Sigma \rightarrow \mathbb{C}$ is measurable. Define the operator $A$ on $L^{p}(\Sigma)$ by

$$
(A x)(\tau):=M(\tau) x(\tau), \quad D(A):=\left\{x \mid x, M x \in L^{p}(\Sigma)\right\} \quad \forall \tau \in \Omega .
$$

Assume the spectrum of $A$, which is the essential image of $M$, is disjoint from $E(\alpha, \beta)$ for some $\alpha, \beta>0$. Then for $\lambda \in \sigma(A)$ with $\operatorname{Re} \lambda>\beta$ we have $|\operatorname{Im} \lambda| \geq e^{\alpha \operatorname{Re} \lambda}$. This implies that, for given real $\lambda_{0}>\beta$ and every $t \in[0, \alpha]$,

$$
\sup \left\{\left|\lambda_{0}-\lambda\right|^{-1} e^{\operatorname{Re} \lambda t} \mid \lambda \in \sigma(A)\right\}<\infty .
$$

Therefore

$$
[W(t) x](\tau):=\left(\lambda_{0}-M(\tau)\right)^{-1} e^{M(\tau) t} x(\tau) \quad \forall t \in[0, \alpha]
$$

defines a local $\left(\lambda_{0}-A\right)^{-1}$-regularized semigroup $\{W(t)\}_{t \in[0, \alpha]}$ generated by $A$. Thus $A$ generates a $\left(\lambda_{0}-A\right)^{-k}$-regularized semigroup $\left\{W_{k}(t)\right\}_{t \in[0, k \alpha]}$ (see [17, Theorem 2.6] for details), or equivalently, $A$ generates a local $k$ times integrated semigroup $\left\{S_{k}(t)\right\}_{t \in[0, k \alpha]}$ and (ACP, $\left.k \alpha\right]$ has a unique solution for all $x \in D\left(A^{k}\right)$ by Theorem 4.4.

Our proof of Example 5.1(a) is more straightforward than that of [2, Example 4.4(a)].

(b) If $\Omega=[0, \infty)$, equipped with the Lebesgue measure, and $M(\tau):=$ $\tau+i e^{\tau}$, then $A$, defined in (5.1), is not densely defined when $p=\infty$.

Example 5.2. (a) [14]. Let $0 \leq p \leq \infty$ and let $0<T<\infty$. Define

$$
\begin{aligned}
& a_{n}:=\frac{n}{T}+i\left\{\left(\frac{e^{n}}{n}\right)^{2}-\left(\frac{n}{T}\right)^{2}\right\}^{1 / 2} \quad \forall n \in \mathbb{N}, \\
& A x:=\left\{a_{n} \xi_{n}\right\}, \quad \text { where } x=\left\{\xi_{n}\right\} \in l^{p} \text { and }\left\{a_{n} \xi_{n}\right\} \in l^{p} .
\end{aligned}
$$


It is easy to see that every $\lambda \geq 0$ is in $\varrho(A)$. In particular, $0 \in \varrho(A)$. Define

$$
W(t):=(-A)^{-1} e^{t A} \quad \forall t \in[0, T) .
$$

For every given $t \in[0, T)$, from

$$
\|W(t)\|=\max \left\{n e^{n t / T} / e^{n}\right\}<\infty,
$$

$\{W(t)\}_{t \in[0, T)}$ is a local $(-A)$-regularized semigroup generated by $A$. From [17, Theorem 2.6], it follows that $A$ generates a local $(-A)^{k}$-regularized semigroup $\left\{W_{k}(t)\right\}_{t \in[0, k T)}$, or equivalently, by Theorem 4.4, $A$ generates a local $k$-times integrated semigroup $\left\{S_{k}(t)\right\}_{t \in[0, k T)}$.

(b) Define

$$
\begin{aligned}
b_{n} & :=\frac{n}{T}+i\left[e^{2 n}-\left(\frac{n}{T}\right)^{2}\right]^{1 / 2} \quad \forall n \in \mathbb{N}, \\
B x & :=\left\{b_{n} \xi_{n}\right\}, \quad \text { where } x=\left\{\xi_{n}\right\} \in l^{p} .
\end{aligned}
$$

As in $(\mathrm{a}), 0 \in \varrho(B)$. Define

$$
\widetilde{W}(t):=(-B)^{-1} e^{t B} \quad(t \in[0, T]) .
$$

For every $t \in[0, T]$, from

$$
\|\widetilde{W}(t)\|=\max \left\{e^{n t / T} / e^{n}\right\}<\infty,
$$

$\{\widetilde{W}(t)\}_{t \in[0, T]}$ is a local $(-B)$-regularized semigroup generated by $B$. If $t>T$, then (5.2) implies that $\|\widetilde{W}(t)\|=\infty$ and hence $\widetilde{W}(t)$ is undefined.

Similarly, $B$ generates a $(-B)^{k}$-regularized semigroup and a $k$-times integrated semigroup by [17, Theorem 2.6] and Theorem 4.4, respectively.

Our proof of Example 5.2 is much easier than that of the example in [14, p. 76].

In the following we present two differential operators generating local regularized semigroups (see $[8,10]$ ).

EXAmple 5.3. (a) Let $1 \leq p<\infty, X$ be one of the spaces $L^{p}\left(\mathbb{R}^{n}\right)$, $\mathrm{BUC}\left(\mathbb{R}^{n}\right)$ and $C_{0}\left(\mathbb{R}^{n}\right)$, or any space where translation is strongly continuous and uniformly bounded. We write $D_{j}:=i \partial / \partial x_{j}(j=1, \ldots, n)$, $D:=\left(D_{1}, \ldots, D_{n}\right)$ and $D^{\alpha}:=\left(D_{1}\right)^{\alpha_{1}} \ldots\left(D_{n}\right)^{\alpha_{n}}$, where $\alpha_{j} \in \mathbb{N} \cup\{0\}$ for $j=1, \ldots, n$.

Let $\mathcal{M}:=\left(p_{i, j}\right)_{i, j=1}^{m}$ be an $m \times m$ matrix of polynomials and let

$$
\mathcal{N}:=\max _{i, j}\left\{\text { degree of } p_{i, j}\right\} .
$$

Define the operator $\mathcal{M}(D):=\left(p_{i, j}(D)\right)$ on $X^{m}$ with $D(\mathcal{M}(D)):=D\left(\Delta^{l}\right)^{m}$ where $l:=1+\frac{1}{2}\left(\mathcal{N}+\frac{n}{2}\right)$. From [8, Theorems 13.9 and 14.1], $\mathcal{M}(D)$ is closable. Set

$$
A:=-\overline{\mathcal{M}(D)}
$$


Then there exists an injective $C \in B(X)$ with dense range such that $-A$ is a subgenerator of a $C$-regularized semigroup $\{W(t)\}_{t \in[0, \infty)}$.

Given $T>0$, define $\widetilde{C}:=W(T)$ and $\widetilde{W}_{k}(t):=W(k T-t)$. Then it is easy to show that $A$ is a subgenerator of the local $\widetilde{C}^{k}$-regularized semigroup $\left\{\widetilde{W}_{k}(t)\right\}_{t \in[0, k T]}$.

(b) The matrix $\mathcal{M}$ of polynomials in (a) is Petrovskiu correct if there exists $\omega \in \mathbb{R}$ such that

$$
\sigma(\mathcal{M}(s)) \subseteq\{z \in \mathbb{C} \mid \operatorname{Re} z \leq \omega\} \quad \forall s \in \mathbb{R}^{n} .
$$

Again from the above references, if $\mathcal{M}$ is Petrovskil correct then there exists $r \geq 0$ such that $-A$ in (5.3) is a subgenerator of an exponentially bounded $(1+\Delta)^{-r}$-regularized semigroup. Given $T>0$, as in (a) of this example, $A$ is a subgenerator of a local regularized semigroup defined on $[0, k T)$ for every $k \in \mathbb{N}$. Simple special cases of (b) are the wave equation (see [10, Example 4.11]) and the equation describing sound propagation in a viscous gas (see [10, Example 4.12]).

\section{References}

[1] W. Arendt, Vector-valued Laplace transforms and Cauchy problems, Israel J. Math. 59 (1987), 327-352.

[2] W. Arendt, O. El-Mennaoui and V. Keyantuo, Local integrated semigroups: Evolution with jumps of regularity, J. Math. Anal. Appl. 186 (1994), 572-595.

[3] G. Da Prato, Semigruppi regolarizzabili, Ricerche Mat. 15 (1966), 223-248.

[4] E. B. Davies and M. M. Pang, The Cauchy problem and a generalization of the Hille-Yosida theorem, Proc. London Math. Soc. 55 (1987), 181-208.

[5] J. A. Goldstein, Semigroups of Linear Operators and Applications, Oxford Univ. Press, 1985.

[6] E. Hille and R. S. Phillips, Functional Analysis and Semigroups, Colloq. Publ. 31, Amer. Math. Soc., 1957.

[7] S. G. Krĕn, G. I. Laptev and G. A. Tsvetkova, On Hadamard correctness of the Cauchy problem for an evolution equation, Soviet Math. Dokl. 11 (1970), 763-766.

[8] R. deLaubenfels, Existence Families, Functional Calculi and Evolution Equations, Lecture Notes in Math. 1570, Springer, 1994.

[9] R. deLaubenfels, Z. Y. Huang, S. W. Wang and Y. Wang, Laplace transforms of polynomially bounded vector-valued functions and semigroups of operators, Israel J. Math. 98 (1997), 189-207.

[10] R. deLaubenfels, G. Z. Sun and S. W. Wang, Regularized semigroups, existence families and the abstract Cauchy problem, Differential Integral Equations 8 (1995), 1477-1496.

[11] F. Neubrander, Integrated semigroups and their applications to the abstract Cauchy problem, Pacific J. Math. 135 (1988), 111-155.

[12] A. Pazy, Semigroups of Linear Operators and Applications to Partial Differential Equations, Springer, New York, 1983. 
[13] N. Sanekata, Some remarks on the abstract Cauchy problem, Publ. Res. Inst. Math. Sci. Kyoto Univ. 11 (1975), 51-65.

[14] N. Tanaka and N. Okazawa, Local C-semigroups and local integrated semigroups, Proc. London Math. Soc. 61 (1990), 63-90.

[15] S. W. Wang, Mild integrated C-existence families, Studia Math. 112 (1995), 251266.

[16] —, Quasi-distribution semigroups and integrated semigroups, J. Funct. Anal. 146 (1997), 352-381.

[17] S. W. Wang and M. C. Gao, Automatic extensions of local regularized semigroups and local regularized cosine functions, Proc. Amer. Math. Soc. 127 (1999), 16511663.

[18] D. V. Widder, An Introduction to Transform Theory, Acad. Press, New York, 1971.

Department of Mathematics

Nanjing University

Nanjing, Jiangsu 210093

P.R. China

E-mail: wang2598@netra.nju.edu.cn

Received July 6, 2001

Revised version February 4, 2002 